\title{
A Quantum Defect Sees its Charged Surroundings
}

\author{
Nitrogen-vacancy centers in diamond are found to be more affected by local charge than \\ expected, which has implications for the use of the defects as quantum sensors.
}

\section{by Ania Bleszynski Jayich*}

$\mathrm{P}$ oint defects in solids are emerging as powerful quantum elements for various technologies, such as sensing, computing, and communication. These defects can not only have long quantum coherence times, but they can also be produced in a scalable way and integrated into devices. Still, researchers must grapple with the fact that, unlike atoms in a vacuum, defects in a solid experience an environment that is, to some extent, uncontrollable and "noisy," which may lead to quantum

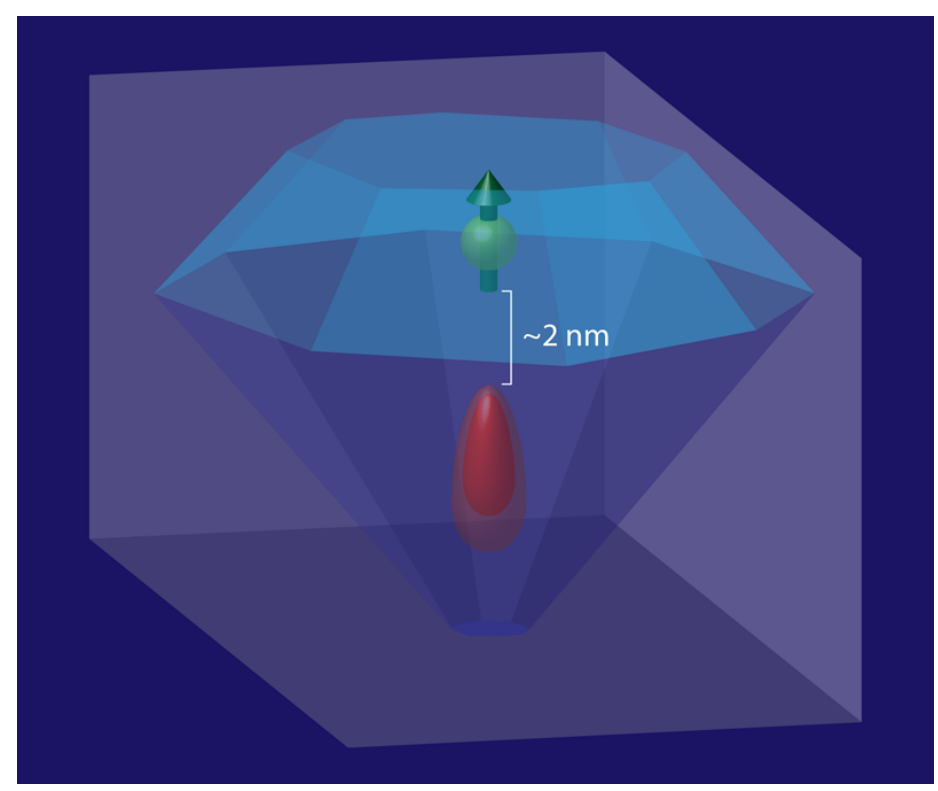

Figure 1: Yao and colleagues harnessed the electric-field sensitivity of an NV center (green arrow) in diamond to locate an electric charge in the defect's vicinity [1]. The dark and light red regions indicate the observed location of the charge with $60 \%$ and $80 \%$ confidence, respectively. (APS/Alan Stonebraker)

\footnotetext{
*Physics Department, University of California, Santa Barbara, CA, USA
}

decoherence of the defect and other unwanted effects. Experiments by Norman Yao of the University of California, Berkeley, and colleagues provide a new level of understanding about environmental effects on a promising kind of defect, the nitrogen-vacancy (NV) center in diamond. The team found that this defect's properties are much more sensitive to electric fields from local charges than previously assumed [1]. This realization may lead to new ways of mitigating, or even harnessing, the environment to enhance the sensing or computing performance of the defects.

An NV center occurs in diamond when nitrogen atoms and vacancies are introduced into the carbon lattice and a nitrogen atom sits next to a vacant site. The ground state of this atom-like defect has three possible electronic spin states, denoted $|+1\rangle,|0\rangle$, and $|-1\rangle$, which give rise to an optically accessible spin-state spectrum. The shape of this spectrum has proven to be highly sensitive to the local magnetic field, electric field, lattice strain, or temperature, and, to date, most of the impressive results with NV centers have been based on this field sensitivity. Researchers have, for example, used the spectral features to detect a single electric charge, image an electron spin, measure temperature variations of $1 \mathrm{mK}$, and determine strain with $10^{-6}$ resolution [2]. Moreover, if nanometer spatial resolution is not essential, then the spectra of large numbers of NV spins can be averaged together to enhance the sensitivity of a measurement. Recently, researchers have also investigated the possibility of harnessing correlations between the defects to increase the sensitivity of ensemble measurements [3].

Spectra of NV centers are measured with the technique of optically detected magnetic resonance (ODMR), in which light from an optically excited NV center is detected while resonantly exciting the defect's spin transitions with microwaves. For field sensing, the linewidths of the spin transitions are key: the narrower the linewidths, the easier it is to resolve small, field-induced line shifts. Unfortunately, any spatial or temporal inhomogeneity in the local environments of the spins will broaden the linewidths. Inhomogeneity is particularly problematic in high-density ensembles, where the introduction of a lot of nitrogen atoms and vacancies into the diamond leads to multiple defects in addition to the desired NV centers. Line shifts and broadening can, for 
example, be caused by magnetic fields from single nitrogen atoms and clusters of vacancies, as well as by strain associated with these defects. These internal sources need to be reckoned with if researchers want to use NV centers to sense fields from external sources.

Yao and colleagues have turned their sights to a largely overlooked inhomogeneity-that of internal electric fields produced by local charges within the diamond. The effect of these internal electric fields hasn't been studied much because most experiments with NV centers are conducted in a magnetic field (to simplify the spectrum), and even a modest magnetic field suppresses the NV's sensitivity to electric fields. In their experiment, Yao and co-workers used very low magnetic fields while analyzing the magnetic resonance spectrum of an ensemble of NV centers. In principle, this spectrum should have two overlapping peaks from the two allowed spin transitions with roughly the same energy, $|0\rangle \rightarrow|+1\rangle$ and $|0\rangle \rightarrow|-1\rangle$. But Yao and colleagues observe a strong splitting of these peaks, as well as a large asymmetry in the line shapes and an unusually sharp feature between the peaks. This splitting has been observed in the past, and it has normally been attributed to local strain. But Yao and co-authors undertake a more careful analysis and point out that strain isn't consistent with the observed asymmetry nor with the lack of a shift in the mean energy of the split peaks.

The researchers are, however, able to explain both of these experimental observations with a model that invokes electric fields from local charges. A key aspect of their model is that, in the absence of a magnetic field, an electric field transverse to the defect's axis (the line from the nitrogen to the vacancy) has a much larger effect on the NV spectrum than a longitudinal field [4]; this asymmetry doesn't occur for strain fields. Finally, they argue that the observed sharp feature is associated with two new states, which are mixtures of the $|+1\rangle$ and $|-1\rangle$ states and are more immune to the decoherence effects of magnetic-field noise [5].

The researchers went a step further and showed they could select one NV center from the ensemble and pinpoint the location of the dominant charge in its vicinity. Here, they measured the chosen NV center's spectrum with polarized microwaves and then analyzed the spectrum's shape to determine the orientation of the local electric field (Fig. 1). In this way, they were then able to locate the dominant charge sitting nanometers away from the NV center with subnanometer-scale accuracy.

Recognizing that charges can dominate the NV spectrum at low magnetic field is the first step to mitigating their unde- sired effects, which can be accomplished through, for example, the preparation of the host material and in situ control of the charges. But many questions remain. What are these charges? Substitutional nitrogen defects are the most obvious suspects, though this possibility isn't consistent with all of the observations made by Yao and colleagues, and further studies are needed. Might the presence of charges also explain why the shape of the NV-center excited-state spectrum has been observed to drift over time in experiments [6]? If the charges move, they would produce fluctuating electric fields that distort the spectrum and contribute to decoherence of the NV centers [7]. But whether the charges fluctuate and, if so, over what timescale, remains unclear. With these unknowns, removing these charges may be the best way to ensure a clean, stable spectrum. Still, one could imagine employing these charges to enhance sensing. One idea to explore is using the lattice charges as "amplifiers" of external electric fields, much like the proposed use of spins as amplifiers for external magnetic fields [8].

This research is published in Physical Review Letters.

\section{REFERENCES}

[1] T. Mittiga et al., "Imaging the local charge environment of nitrogen-vacancy centers in diamond," Phys. Rev. Lett. 121, 246402 (2018).

[2] R. Schirhagl, K. Chang, M. Loretz, and C. L. Degen, "Nitrogenvacancy centers in diamond: Nanoscale sensors for physics and biology," Ann. Rev. Phys. Chem. 65, 83 (2014).

[3] S. Choi, N. Yao, and M. Lukin, "Quantum metrology based on strongly correlated matter," arxiv:1801.00042.

[4] E. Van Oort and M. Glasbeek, "Electric-field-induced modulation of spin echoes of $\mathrm{N}-\mathrm{V}$ centers in diamond," Chem. Phys. Lett. 168, 529 (1990).

[5] F. Dolde et al., "Electric-field sensing using single diamond spins," Nat. Phys. 7, 459 (2011).

[6] V. M. Acosta et al., "Dynamic stabilization of the optical resonances of single nitrogen-vacancy centers in diamond," Phys. Rev. Lett. 108, 206401 (2012).

[7] B. A. Myers, A. Ariyaratne, and A.C. Bleszynski Jayich, "Double-quantum spin-relaxation limits to coherence of nearsurface nitrogen-vacancy centers," Phys. Rev. Lett. 118, 197201 (2017).

[8] M. Schaffry, E. M. Gauger, J. J. L. Morton, and S. C. Benjamin, "Proposed spin amplification for magnetic sensors employing crystal defects," Phys. Rev. Lett. 107, 207210 (2011).

10.1103/Physics.11.126 